\title{
Cilostazol for Aneurysmal Subarachnoid Hemorrhage: An Updated Systematic Review and Meta-Analysis
}

\author{
Jiangfeng Liu ${ }^{\mathrm{a}, \mathrm{b}}$ Jingli He ${ }^{\mathrm{a}}$ Xun Chen ${ }^{\mathrm{a}}$ Yanjin Feng ${ }^{\mathrm{a}}$ Chen Wang ${ }^{\mathrm{a}}$ \\ Mohamed Arab Awil ${ }^{\mathrm{a}}$ Yan Wang ${ }^{\mathrm{c}}$ Yi Tian ${ }^{\mathrm{d}}$ Deren Hou ${ }^{\mathrm{a}}$ \\ aDepartment of Neurology, The Third Xiangya Hospital, Central South University, Changsha, China; bepartment \\ of General Practice, Zhongshan People's Hospital of Guangdong Province, Zhongshan, China; 'Department of

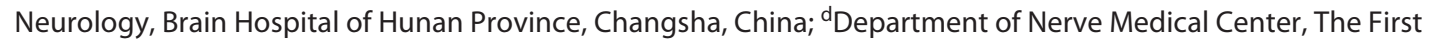 \\ Hospital of Changsha, Changsha, China
}

\section{Keywords}

Cilostazol · Aneurysm · Subarachnoid hemorrhage ·

Meta-analysis

\begin{abstract}
Background and Purpose: Delayed cerebral ischemia is a major cause of morbidity and mortality in patients with aneurysmal subarachnoid hemorrhage (aSAH). Cilostazol, a selective inhibitor of phosphodiesterase 3, was reported to reduce cerebral vasospasm and improve outcomes. We aimed to conduct an updated systematic review and meta-analysis of the efficacy and safety of cilostazol in aSAH. Methods: We systematically searched PubMed, Embase, MEDLINE, and the Cochrane Library for articles published in English with the latest publishing time in August 2020. Articles reporting favorable outcome as the primary outcome and reporting severe angiographic vasospasm (aVS), symptomatic vasospasm (sVS), new cerebral infarction, or mortality as the secondary outcome were included in this review. Furthermore, we examined whether clinical outcomes were associated with the dosage of cilostazol ( $300 \mathrm{mg} /$ day vs. $100-200 \mathrm{mg} /$ day). Results: Data from 405 patients in 4 randomized con-
\end{abstract}

karger@karger.com

(C) 2022 S. Karger AG, Basel

www.karger.com/ced

Karger $\stackrel{2}{*}$ trolled trials (RCTs) and 461 patients in 4 observational studies (OSs) were included. In RCT studies, cilostazol was associated with significant favorable outcomes at discharge or 1 month (risk ratio [RR] 1.41, 95\% confidence interval [CI] 1.01$1.97, p=0.04$ ) or 3 or 6 months (RR $1.16,95 \% \mathrm{Cl} 1.05-1.28$, $p=0.002$ ). However, in OSs, no significant difference was indicated in favorable outcomes at discharge or 1 month (RR $1.22,95 \% \mathrm{Cl} 0.94-1.60, p=0.14$ ) nor 3 or 6 months (RR 1.29, $95 \% \mathrm{Cl} 0.92-1.81, p=0.14)$. The analyses found that cilostazol significantly reduced the incidences of severe aVS (RCT: RR 0.64, 95\% Cl 0.41-1.01, $p=0.05$; OS: RR $0.61,95 \% \mathrm{Cl}$ $0.43-0.88, p=0.007$ ), sVS (RCT: RR $0.46,95 \% \mathrm{Cl} 0.31-0.70$, $p=0.0002 ;$ OS: $\mathrm{RR} 0.38,95 \% \mathrm{Cl} 0.21-0.68, p=0.001)$, and new cerebral infarction (RCT: RR 0.40, 95\% Cl 0.24-0.67, $p=0.0005$; OS: RR $0.38,95 \% \mathrm{Cl} 0.23-0.64, p=0.0002)$. However, no significant difference in mortality (RCT: RR $0.86,95 \% \mathrm{Cl} 0.23-$ $3.21, p=0.82$; OS: RR $0.16,95 \% \mathrm{Cl} 0.02-1.24, p=0.08$ ) was found. In 3 OSs which reported different doses of cilostazol ( $300 \mathrm{mg} /$ day vs. $100-200 \mathrm{mg} /$ day) for aSAH, the $300-\mathrm{mg} /$ day cilostazol groups showed decreased delayed cerebral infarction (RR $0.27,95 \% \mathrm{Cl} 0.09-0.81, p=0.02$ ) but no significant difference in shunt-dependent hydrocephalus (RR 0.92, 95\% $\mathrm{Cl} 0.33-2.60, p=0.88$ ) or functional outcomes (RR $1.14,95 \%$ 
$\mathrm{Cl} 0.74-1.75, p=0.56)$ compared with the $100-200 \mathrm{mg} /$ day cilostazol groups. Conclusions: The meta-analyses suggest the credible efficacy and safety of cilostazol in treating aSAH. Furthermore, 300-mg/day cilostazol treatment appeared to be more effective than 100-200 mg/day treatment.

(c) 2022 S. Karger AG, Basel

\section{Introduction}

Aneurysmal subarachnoid hemorrhage $(\mathrm{aSAH})$ is a neurological emergency with considerable morbidity and mortality. It accounts for up to 5\% of strokes each year and usually occurs at a relatively young age, with higher incidence in women, leading to a high loss of potential life years [1,2]. The 2 most important causes of mortality of subarachnoid hemorrhage are delayed cerebral ischemia (DCI) and early brain injury [3]. DCI is defined as any neurological deterioration presumed related to ischemia that persisted for $\geq 1 \mathrm{~h}$, independent of imaging findings, after rigorous exclusion of other potential causes of clinical deterioration. The pathophysiology of DCI is postulated to include an interaction of delayed onset of angiographic vasospasm (aVS), impaired autoregulation, microthrombosis, capillary transit time heterogeneity, and cortical spreading ischemia [3]. aVS is considered to be the main cause of DCI $[2,3]$. Currently, nimodipine, as a calcium channel blocker, is the most commonly used drug for the treatment of DCI in many countries [1]. However, it does not reduce the rate of vasospasm even though it improves outcomes $[4,5]$.

Some animal experiments [6-8] and clinical studies [9-16] have suggested that cilostazol improves vasospasm of aSAH in recent years. Cilostazol is a selective inhibitor of phosphodiesterase 3, which in turn increases the activation of intracellular cAMP and thereby inhibits platelet aggregation and exerts vasodilatory and anti-inflammatory effects [17]. Prior systematic reviews examining cilostazol treatment had limitations as they included different doses of cilostazol and pooling results with diverse follow-up times but had not analyzed the effect of dosage and follow-up time on patients' outcomes [1820]. In this study, we conducted a meta-analysis on the complete results from recently published clinical trials to assess the association between cilostazol and clinical outcomes, including favorable outcomes, severe aVS, symptomatic vasospasm (sVS), new cerebral infarction, and mortality among patients with aSAH. We aimed to examine whether clinical outcomes were associated with the dosage of cilostazol.

Cilostazol for Aneurysmal Subarachnoid Hemorrhage

\section{Methods}

\section{Search Strategy}

We undertook a systematic review and meta-analysis based on a predefined protocol in accordance with the International Platform of Registered Systematic Review and Meta-analysis Protocols. We searched PubMed, Embase, MEDLINE, and the Cochrane Library for these keywords: "Subarachnoid Hemorrhage, aneurysm, SAH, cilostazol." Boolean operators were used as appropriate (see the Search Strategy in the Supplement). We restricted our inclusion of articles to those written in English. The latest articles searched were published in August 2020.

\section{Study Selection}

Two investigators (Liu J.F. and He J.L.) independently selected and evaluated studies according to the eligibility criteria. Disagreements between the investigators concerning the decision to include or exclude a study were resolved by consultation with the senior author (Hou D.R.). Eligible trials had to meet the following criteria: (1) the study must be randomized controlled trials (RCTs) or observational studies (OSs); (2) the study must include a cilostazol group and a control group. The patients received mainly fasudil hydrochloride treatment in the control group and cilostazol and fasudil treatment in the cilostazol group; (3) the study must report events of interest in each group, such as favorable outcomes, or severe aVS; and (4) the patients in the studies must be adult aSAH patients ( $\geq 18$ years). Articles were excluded if they have reported overlapping data or have not reported events of interest. Articles about animal studies, reviews, and comments were also excluded.

\section{Data Extraction}

Two investigators (Liu J.F. and He J.L.) independently extracted the following information from each eligible article: name of the first author, study design, study location (country), study period, follow-up time, treatment modalities, and baseline characteristics of patients (e.g., mean ages of participants, number of participants, surgery type, and severity assessment of participants). Disagreements between the 2 investigators were resolved by consultation with the senior author.

\section{Quality Assessment}

Two investigators (Liu J.F. and He J.L.) independently performed quality assessment. We used Cochrane Collaboration's tool [21] and Newcastle Ottawa Scale (NOS) [22] to assess the risk of bias among the RCTs and OSs, respectively.

\section{Outcome Measures}

The primary outcome was a favorable outcome. The secondary outcomes comprised of severe aVS, sVS, new cerebral infarction, and mortality. The definition of the favorable outcome was the modified Rankin Scale (mRS) score of $0-2$, the Glasgow Outcome Scale (GOS) score of 4-5, or the extended GOS (eGOS) score of 5-8 evaluated at the final follow-up. Severe aVS was defined as greater than $50 \%$ of reduction in the vessel diameter in catheter angiography or CT angiography. sVS was defined as the development of a new focal or global neurological deficit or deterioration of at least 2 points on the Glasgow Coma Scale, which could not be explained by such factors as hydrocephalus, rebleeding, infection, or electrolyte disturbance. New cerebral infarction was assessed 
Fig. 1. Flowchart of the literature search and study selection.

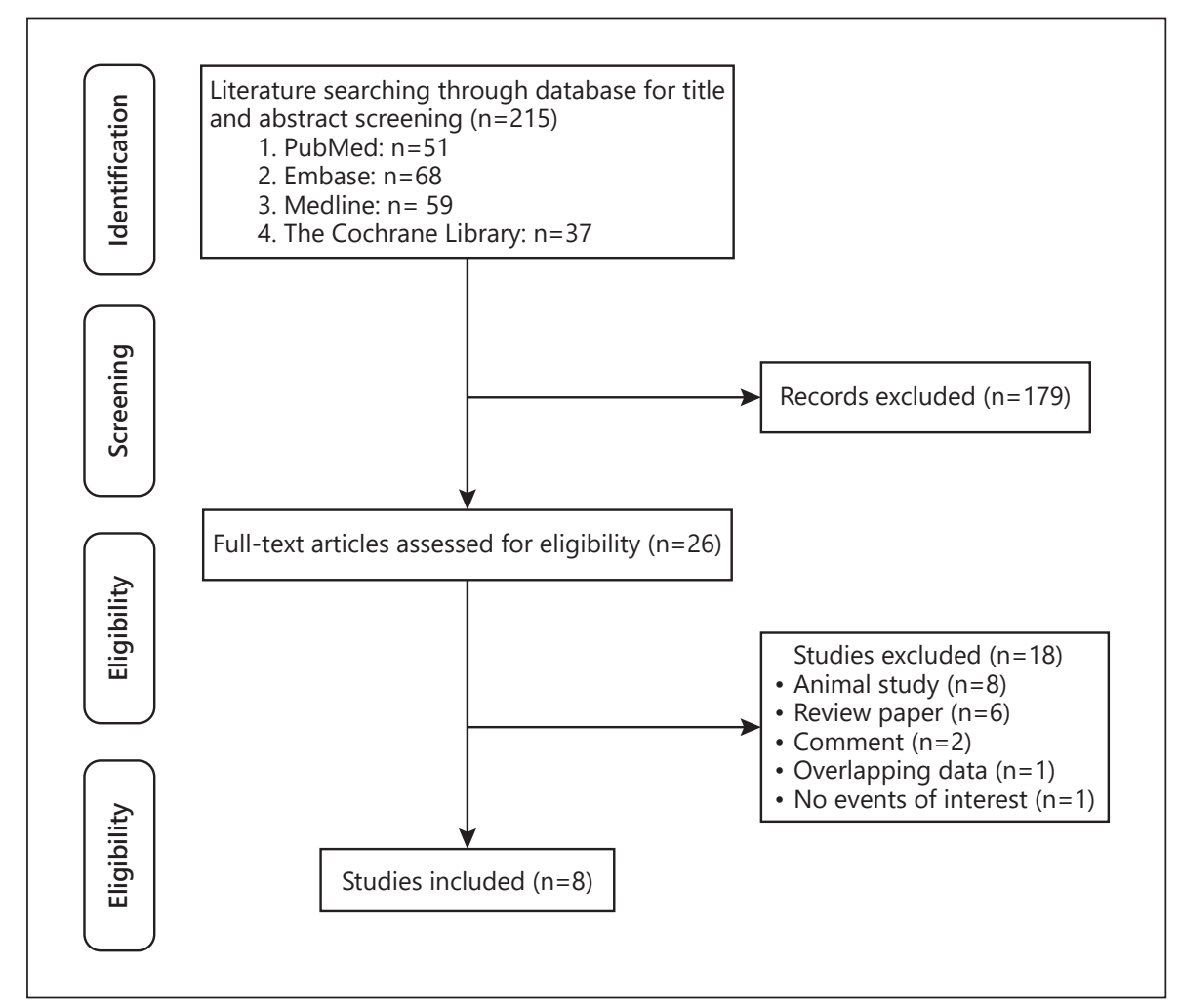

with computed tomography scanning or MRI, excluding procedural-related infarction. Furthermore, we examined whether clinical outcomes such as delayed cerebral infarction, shunt-dependent hydrocephalus, and mRS score (0-2) at discharge were associated with the dosage of cilostazol (300 mg/day vs. 100-200 mg/ day). Delayed cerebral infarction was defined as a new infarct on CT scans or MRI that was not visible on the day following operation or intervention. The definition of shunt-dependent hydrocephalus was persistent and symptomatic hydrocephalus presenting after aSAH that required permanent cerebrospinal fluid (CSF) diversion.

\section{Statistical Analysis}

We calculated and subsequently pooled in independent metaanalyses, risk ratio (RR), and its $95 \%$ confidence interval (CI) for each outcome of interest, with a 2 -sided $p \leq 0.05$, indicating a significant difference. A random-effects model was used in metaanalysis. Subgroup analyses stratified by study types were performed. Heterogeneity across trials was investigated by the Cochran's $Q$ test and measured by the $I^{2}$ statistic, with $I^{2}$ values exceeding 25\%, 50\%, and 75\%, representing low, moderate, and high heterogeneity, respectively [23]. Sensitivity analyses were conducted by sequentially excluding one study at a time to check whether the results were robust. Where data were not given in a way that could be used in the meta-analysis or where only 1 or 2 significant studies were identified for a given biomarker, the findings of these studies are only listed in the Discussion section. All analyses were performed using RevMan (version 5.4).

\section{Results}

\section{Study Characteristics}

Figure 1 shows a flow diagram for the study selection process. We identified 215 studies, of which data from 8 trials [9-16] were used. The 8 trials included 866 patients (479 in the cilostazol group and 387 in the control group) with the male/female ratio of $0.42(254 / 612)$ and the mean age of 62.1 years. The procedures of aneurysm surgery were clipping and coiling in all patients, of whom $76.2 \%$ were treated by clip ligation. All 8 eligible studies were from Japan. They comprised of 4 RCTs $[10,11,13,15]$ and 4 OSs $[9,12,14,16]$ and were all published between 2009 and 2019. In view of the independence between 2 studies, we divided 1 research into 2 independent studies as "Nakatsuka et al. [14] P and R" in the meta-analyses. Finally, 9 eligible studies are shown in Table 1 in this review. Five studies appraised the outcomes at discharge or 1 month, and the other 4 studies reported the 3- or 6-month followup outcomes. The clinical outcomes at different stages were assessed in this review. Analyses were performed on eligible studies with the same dosage of cilostazol (200 mg/ day) in the primary outcome and secondary outcomes. Three studies compared different doses of cilostazol. In-
Liu/He/Chen/Feng/Wang/Awil/Wang/ Tian/Hou 


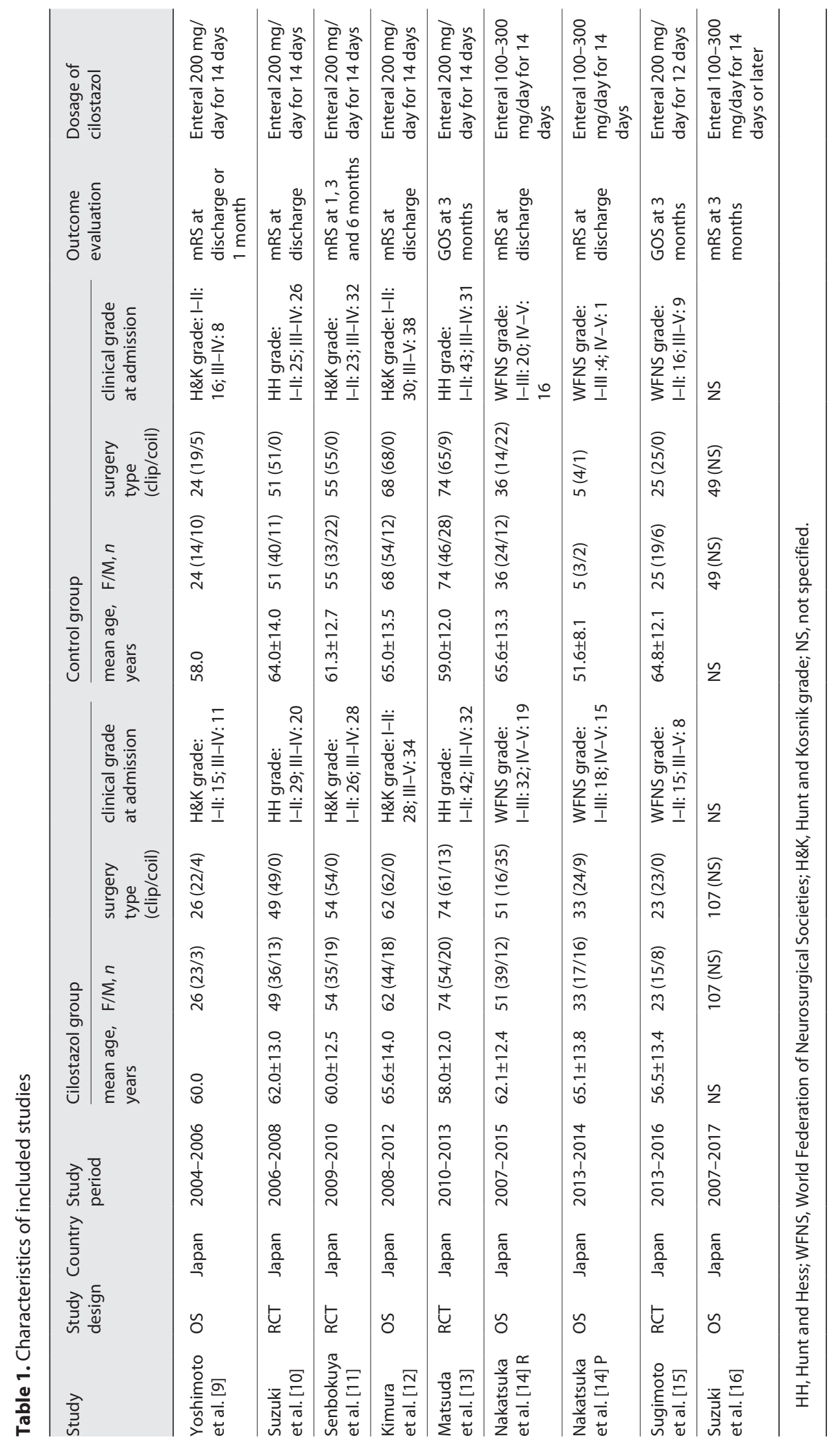


Favorable outcome at two different periods

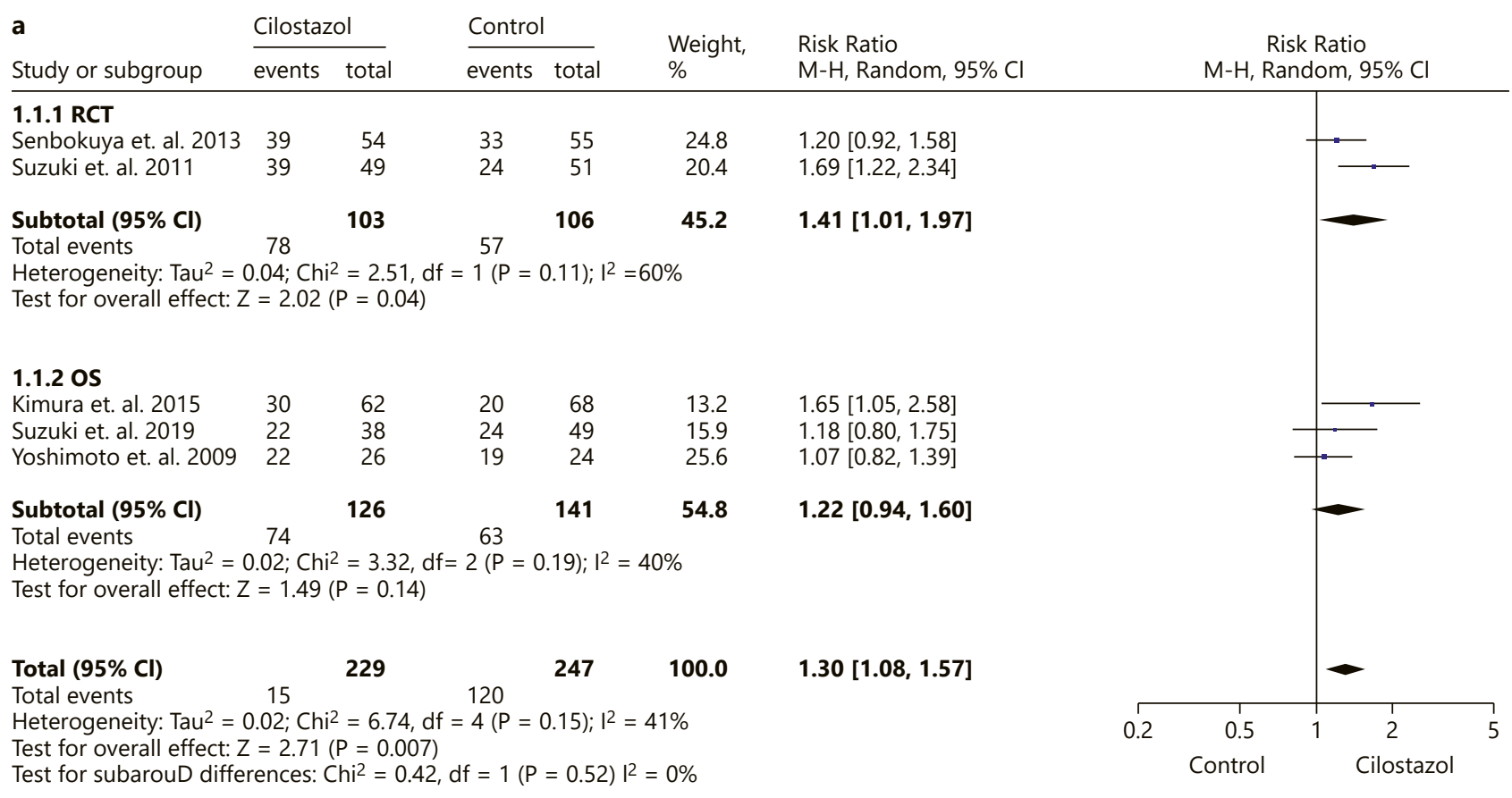

\begin{tabular}{|c|c|c|c|c|c|}
\hline b & Cilostazol & Control & & & Risk Ratio \\
\hline Study or subgroup & $\overline{\text { events total }}$ & events total & & $\mathrm{M}-\mathrm{H}$, Random, $95 \% \mathrm{Cl}$ & $\mathrm{M}-\mathrm{H}$, Random, $95 \% \mathrm{Cl}$ \\
\hline
\end{tabular}

\begin{tabular}{lcccrrr}
\hline $\mathbf{2 . 1 . 1}$ RCT & & & & & & \\
Matsuda et. al. 2016 & 70 & 74 & 61 & 74 & 60.3 & $1.15[1.02,1.29]$ \\
Senbokuya et. al. 2013 & 48 & 54 & 42 & 55 & 27.7 & $1.16[0.98,1.39]$ \\
Sugimoto et. al. 2018 & 17 & 23 & 14 & 25 & 4.7 & $1.32[0.86,2.02]$ \\
& & $\mathbf{1 5 1}$ & & $\mathbf{1 5 4}$ & $\mathbf{9 2 . 7}$ & $\mathbf{1 . 1 6}[\mathbf{1 . 0 5}, \mathbf{1 . 2 8}]$ \\
$\begin{array}{l}\text { Subtotal (95\% Cl) } \\
\text { Total events }\end{array}$ & 135 & & 117 & & &
\end{tabular}

Heterogeneity: $\operatorname{Tau}^{2}=0.00 ; \mathrm{Chi}^{2}=0.44, \mathrm{df}=2(\mathrm{P}=0.80) ; \mathrm{I}^{2}=0 \%$

Test for overall effect: $Z=3.06(P=0.002)$

2.1.2 OS

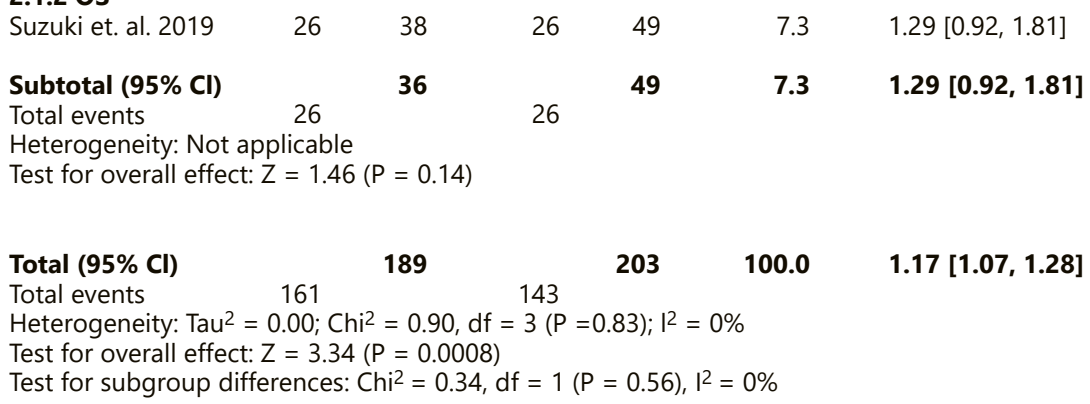

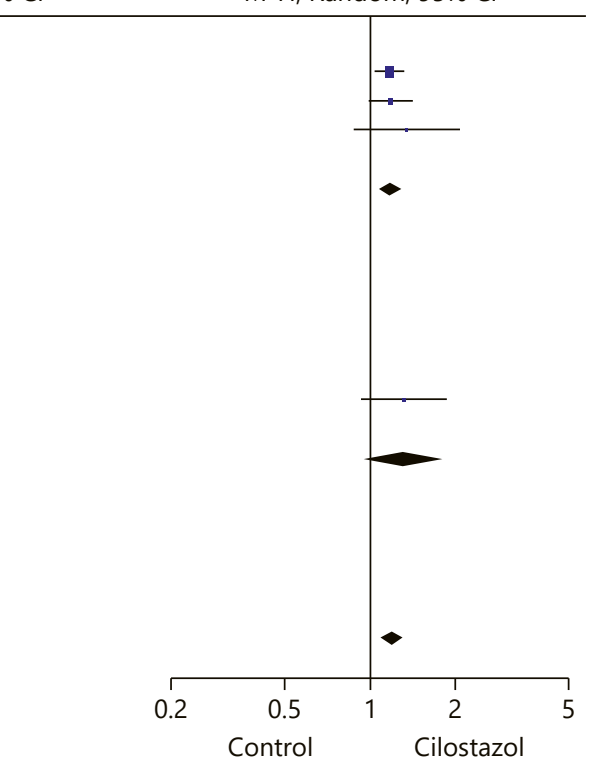

Fig. 2. Favorable outcome at two different periods. a Favorable outcome at discharge or 1 month. b Favorable outcome at 3 months or 6 months. 
cluded trials and patient characteristics are summarized in Table 1 and detailed about diverse dosage in online supplementary Table 1 (for all online suppl. material, see www.karger.com/doi/10.1159/000518731).

\section{Risk of Bias Assessment and Publication Bias}

Of the 4 RCTs, 3 studies showed low risk and the other one moderate risk of bias on Cochrane Collaboration's tool. Overall OSs scored 7 on the NOS (online suppl. Table 2). We did not observe significant publication bias based on the funnel plots for the primary outcome (online suppl. Fig. 1).

\section{Overall Effect on Favorable Outcomes}

Figure 2 presents favorable outcomes at 2 different periods (at discharge or 1 month or 3 months or 6 months). As Senbokuya et al. 2013 and Suzuki et al. [16] reported the functional outcomes using the mRS score at 2 different stages, we used the corresponding data from 2 trials in our study. The meta-analysis of trials with favorable outcomes at discharge or 1 month found statistically significant difference in the RCT (RR 1.41, 95\% CI 1.01$1.97, p=0.04$, Fig. $2 \mathrm{a}$ ) but not in the OS (RR $1.22,95 \%$ CI $0.94-1.60, p=0.14$, Fig. 2a). The heterogeneity result $\left(I^{2}=41 \%, p=0.15\right)$ of the trials was acceptable. The incidence of favorable outcomes at 3 months and 6 months were reported in the RCT with the acceptable overall effect size (RR 1.16, 95\% CI 1.05-1.28, $p=0.002$, Fig. 2b) and heterogeneity $\left(I^{2}=0 \%, p=0.80\right)$, indicating that the cilostazol group was associated with more significant proportional benefit than the control group.

\section{Overall Effect on Secondary Outcomes}

The rate of severe aVS was $23.5 \%$ for the cilostazol group (95\% CI 17.1-28.9\%) and 38.6\% (95\% CI 33.1$44.9 \%$ ) for the control group (RCT: RR $0.64,95 \%$ CI $0.41-$ $1.01, p=0.05$; OS: RR $0.61,95 \%$ CI $0.43-0.88, p=0.007$, Fig. 3a). The rates of sVS showed statistically significant difference between groups: $14.3 \%$ (95\% CI 10.1-17.9\%) for the cilostazol group and 34.2\% (95\% CI, 28.1-39.9\%) for the control group (RCT: RR 0.46, 95\% CI 0.31-0.70, $p=0.0002$; OS: RR 0.38 , 95\% CI $0.21-0.68, p=0.001$, Fig. 3b). The control group was associated with a significantly higher rate of new cerebral infarction $(26.6 \%, 95 \%$ CI $22.7-30.5 \%)$ than the cilostazol group $(10.1 \%$, 95\% CI $6.2-14.0 \%$ ), corresponding to a pooled RR of 0.39 (RCT: RR 0.40, 95\% CI 0.24-0.67, $p=0.0005$; OS: RR 0.38, 95\% CI $0.23-0.64, p=0.0002$, Fig. $3 c$ ). The mortality was $2.6 \%$ in the cilostazol group (95\% CI $0.6-4.6 \%)$ and $6.0 \%$ in the control group (95\% CI 2.1-9.9\%). No significant differ-

Cilostazol for Aneurysmal Subarachnoid Hemorrhage ence in the rate of death was found between the 2 groups (RCT: RR 0.86, 95\% CI 0.23-3.21, $p=0.82$; OS: RR 0.16, $95 \%$ CI $0.02-1.24, p=0.08$, Fig. $3 \mathrm{~d}$ ). There was no significant heterogeneity among these studies in secondary outcomes.

Overall Effect of $300 \mathrm{mg} /$ day versus 100-200 mg/day

Three studies reported different doses of cilostazol (300 mg/day vs. 100-200 mg/day) for aSAH (Nakatsuka et al. [14] P, R, and Suzuki et al. [16]) (Fig. 4, with details in online suppl. Table 1). The $300-\mathrm{mg} /$ day treatment was associated with a decreased delayed cerebral infarction rate $(4.1 \%)$, whereas the delayed cerebral infarction rate was $17.8 \%$ for the $100-200 \mathrm{mg}$ /day treatment (pooled RR 0.27 , 95\% CI 0.09-0.81, $p=0.02$ ). No significant difference in rates of shunt-dependent hydrocephalus between groups was found (RR 0.92, 95\% CI 0.33-2.60, $p=0.88$ ) nor was there significant difference in the rates of treatment benefit across the mRS scores: $53.4 \%$ for $300 \mathrm{mg} /$ day treatment versus $51.7 \%$ for $100-200 \mathrm{mg} /$ day treatment (RR 1.14, 95\% CI 0.74-1.75, $p=0.56$ ). There was no indication of significant heterogeneity among these analyses.

\section{Discussion}

This updated systematic review and meta-analysis reports detailed analyses of 4 RCTs and 4 OSs that compared the cilostazol group and the control group for aSAH patients. The results of this meta-analysis show that cilostazol therapy was associated with improved outcomes at discharge or 1 month as well as at 3 months and 6 months. However, there was no significant difference of favorable outcomes at discharge or 1 month among observational trials. The meta-analysis indicates that cilostazol significantly reduced incidences of aVS, sVS, and new cerebral infarction, as compared with the control group. In addition, no significant differences in the mortality rate were found between groups.

We compared the effects of cilostazol at different dosages on aSAH, which had never been done in previous meta-analyses. The 300-mg/day treatment with cilostazol showed significant efficacy compared with 100-200 mg/ day in decreasing delayed cerebral infarction. In addition, the $300-\mathrm{mg} /$ day therapy demonstrated higher rates of shunt-dependent hydrocephalus and favorable outcomes than the 100-200-mg/day therapy. However, the difference was not statistically significant. All these results suggested that $300 \mathrm{mg} /$ day cilostazol treatment appeared to be

Cerebrovasc Dis 2022;51:138-148 143 
Secondary outcomes in the two groups

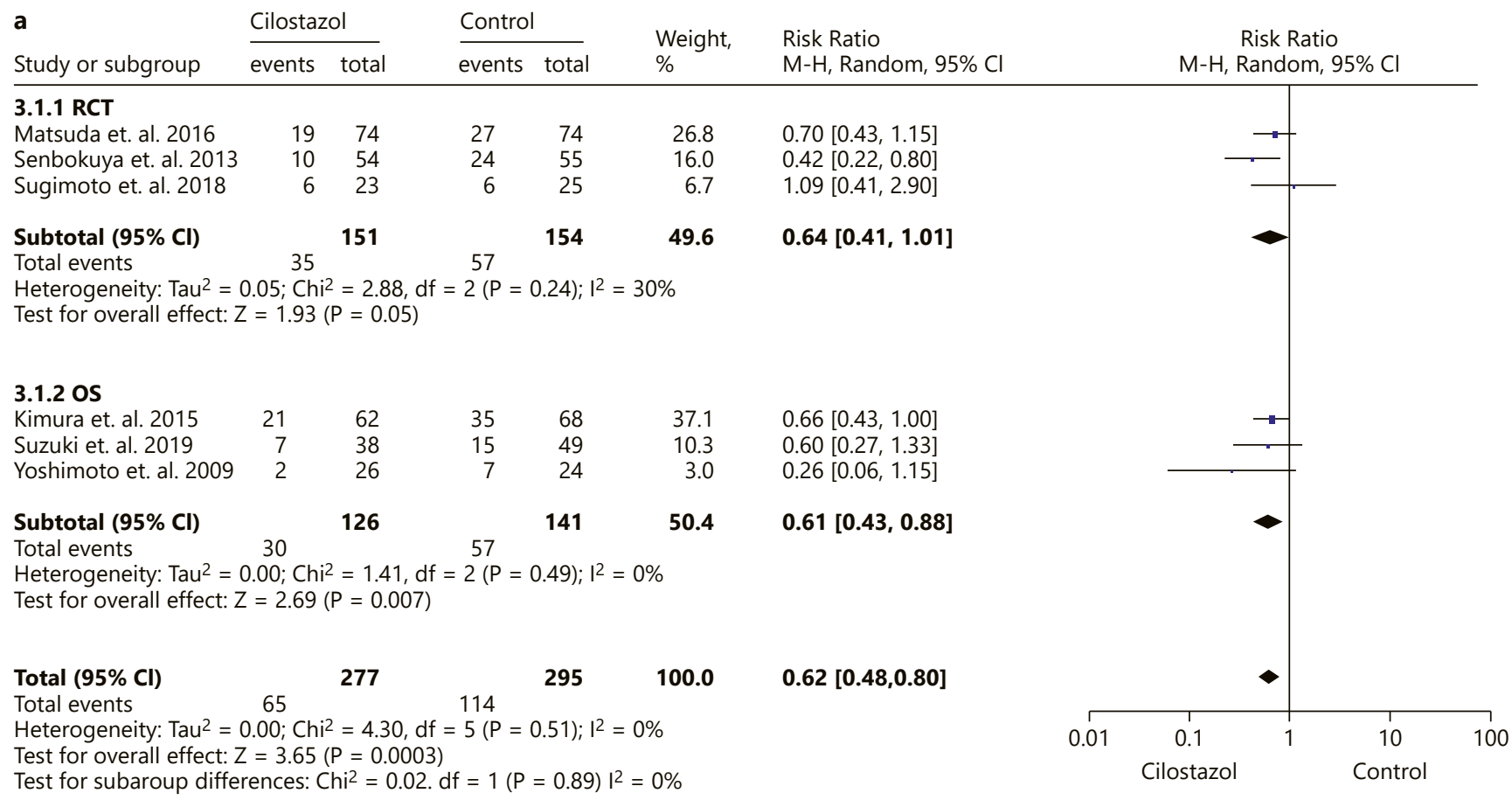

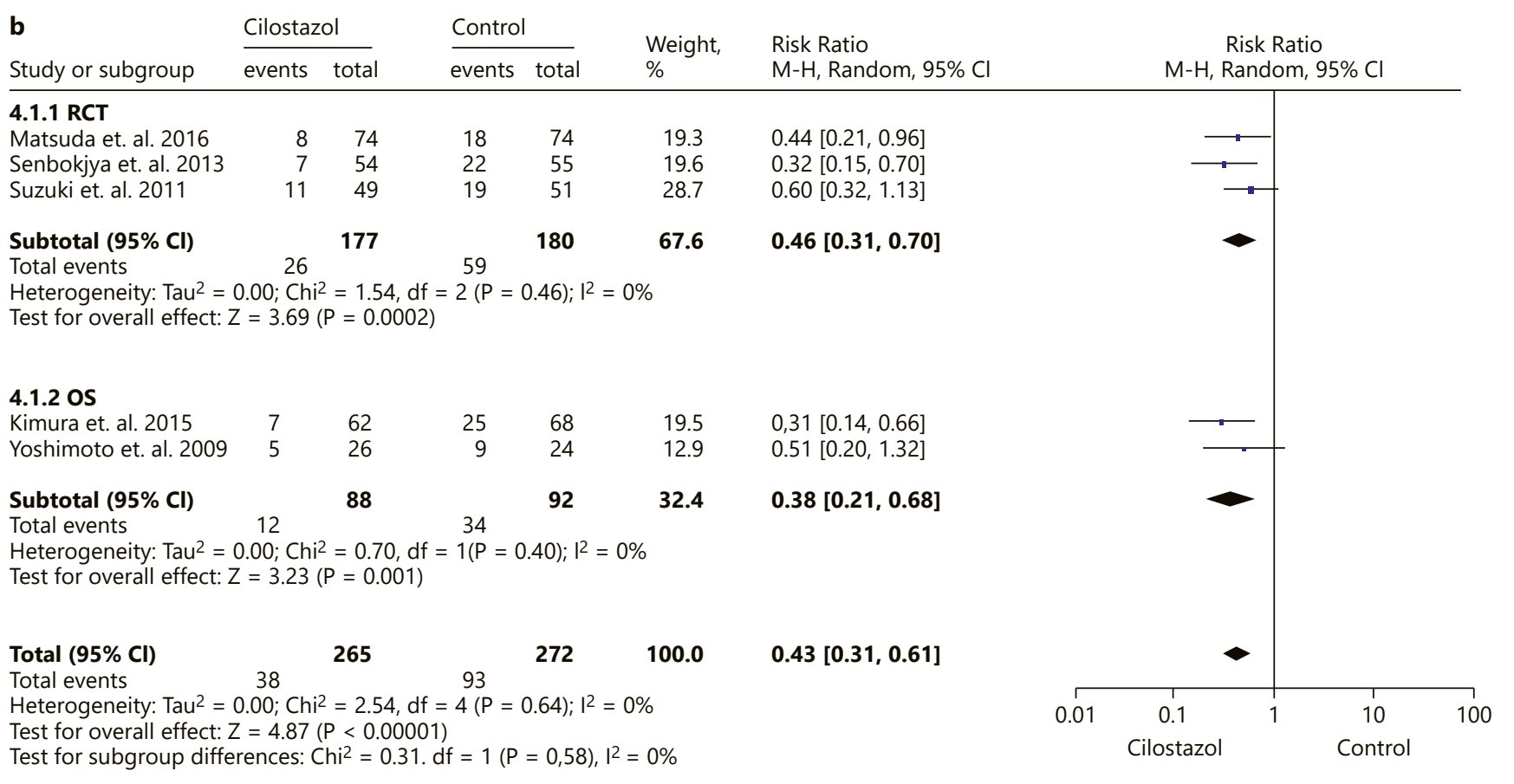

Fig. 3. Secondary outcomes in the two groups. a Severe aVS. b sVS. c New cerebral infarction. d Mortality.

(Figure continued on next page.) 


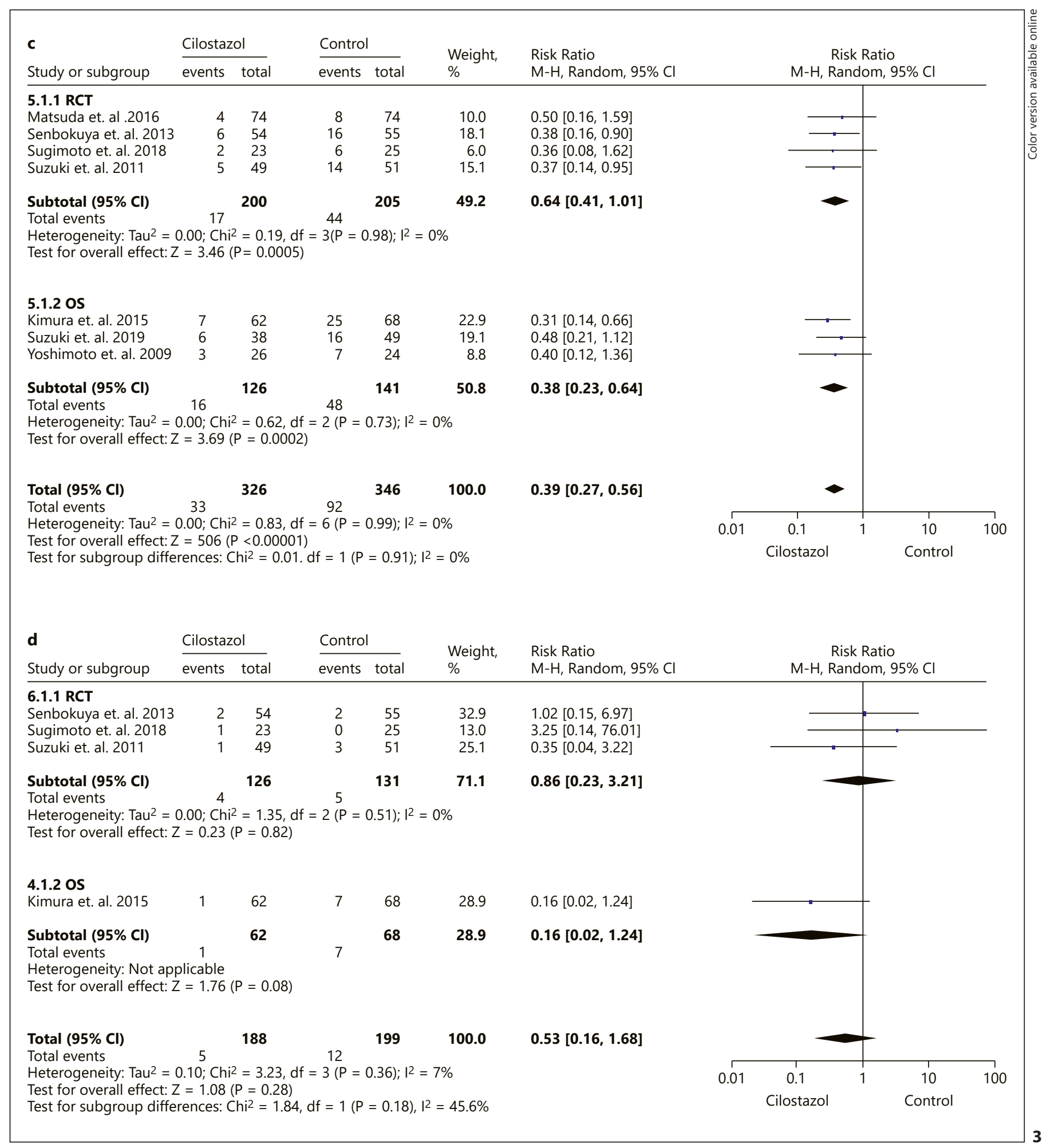

Cilostazol for Aneurysmal Subarachnoid 
Clinical outcomes at different cilostazol doses (300 mg/day vs $100-200 \mathrm{mg} /$ day)

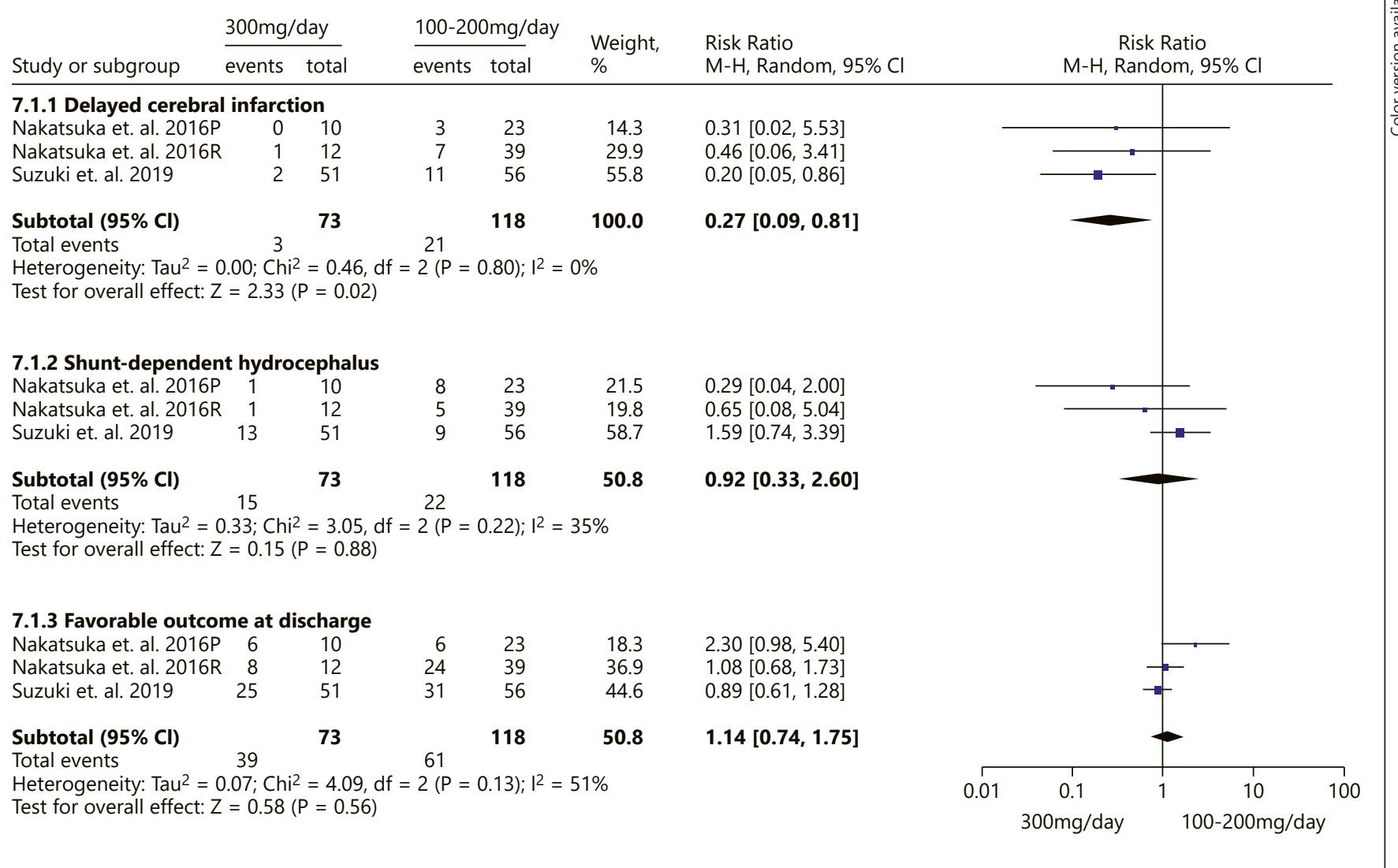

Fig. 4. Clinical outcomes at different cilostazol doses (300 mg/day vs. 100-200 mg/day).

more effective than 100-200 mg/day. However, this metaanalysis included only 3 OSs, which might introduce bias.

Nakatsuka et al. [14] P found that $300 \mathrm{mg} /$ day cilostazol suppressed tenascin-C (TNC) levels more effectively than 100-200 mg/day cilostazol. Suzuki et al. [16] found that the $300 \mathrm{mg} /$ day cilostazol cohort had lower plasma TNC levels and a lower incidence of delayed cerebral infarction with better outcomes than the noncilostazol cohort. TNC, a matricellular protein, is extremely limited in normal adult tissues. Significantly higher TNC levels in the CSF were associated with worse admission clinical grade, aVS, DCI, and worse outcomes [24]. Cilostazol can inhibit TNC induction at the transcriptional level [25]. But, TNC tested in these 2 studies were from the peripheral blood in patients. Although plasma may reflect TNC levels to some extent, no studies have investigated whether peripherally detected TNC levels accurately reflect the levels in CSF [26]. TNC is still promising as a biomarker for noninvasive early diagnosis and mon- itoring of post-SAH brain injuries and as a molecular target for the development of new therapies.

In our analysis, most patients used Fasudil hydrochloride except a few participants in Suzuki et al.'s [10] study. Fasudil is a rho-kinase inhibitor that acts as a vasodilator [17]. Cilostazol has a vasodilatory effect on the cerebral arteries as well by increasing intracellular concentrations of cAMP through an inhibitory effect on phosphodiesterase 3 [17]. The combination effects of these 2 drugs, such as additivity, synergy, and interaction, remain unclear and need further studies. Nimodipine, a notable drug proved to improve neurological outcomes and decrease mortality in patients with aSAH, was not used in the included studies because it had not obtained approval for use in Japan. No convincing evidence has shown that nimodipine affects the incidence of either angiographic or sVS at present [5]. Some researchers have been conducting clinical trials about the combination therapy of nimodipine and cilostazol in aSHA according to the Co- 
chrane Central Register of Controlled Trials. Further studies on the application and effectiveness of the combination of the 2 drugs might yield promising results.

This meta-analysis synthesizes evidence from randomized studies, and observational trials may help inform the design and execution of future studies, examining the efficacy of cilostazol for aSAH. Additional trials are needed to systematically study the relationship between patient-, disease-, and treatment-related factors and outcomes following cilostazol treatment. Perhaps, most importantly, the dosage and treatment course of cilostazol need to be explored. In addition, trials should explore and define the optimal cilostazol therapy with respect to age, clinical grade at admission, location of aneurysms, and the presence of complications, if any. The relationship between these variables and drug safe, as well as mortality and morbidity, should also be studied. These studies could inform the development of new therapy for patients with aSAH.

Our study had several limitations of note. First, although we did not find significant heterogeneity between studies, we failed to consider other inherent differences among the included studies, such as aSAH severity, outcome evaluation type, and surgery type. Each of these factors is a potential confounder. In the patients treated by neurosurgical clipping and endovascular coiling in eligible studies, $76.2 \%$ underwent clipping. Clipping was associated with hypovolemia in the early postoperative stage and with poorer preload responsiveness to volume therapy during the vasospasm risk period compared with endovascular coiling [27]. Therefore, a high proportion of patients who underwent clipping may influence the metaanalysis. Kimura et al. [12] used intensive nutrition support in the cilostazol group, and Suzuki et al. [10] used statin and ozagrel rather than fasudil for a few patients, which were considered main causes of heterogeneity for the favorable outcome at discharge or 1 month. Second, all the included studies were conducted in Japan cohorts. Despite that the outcomes were significantly improved with cilostazol for aSAH in our meta-analysis, the drug has not yet been tested in non-Japan population. Therefore, it is not recommended for patients with aSAH by the current guidelines in all the other countries. Additional large-scale studies are needed to determine whether our findings are valid in non-Japan population. Third, in order to assess the patient's prognosis comprehensively, it is necessary to conduct multivariate analyses on functional outcomes. However, we could not acquire detailed information of functional outcomes due to inaccessibility to patient-level data. Fourth, we restricted our inclusion cri- teria to those written in English in order to ensure the quality of articles, but the limitation might lead to publication bias. Finally, the analyses about different dosages of cilostazol only included 3 observational trials, which may lead to reduced reliability of the results. Further studies on different-dose cilostazol treatments are needed.

\section{Conclusion}

The meta-analyses suggest the credible efficacy and safety of cilostazol in treating aSAH. Furthermore, 300 $\mathrm{mg} /$ day cilostazol treatment appeared to be more effective than 100-200 mg/day treatment.

\section{Acknowledgments}

We thank Langzhong 1987 and Zhirui Zhou from the DXY for the assistance in statistical analysis. We are grateful to Dr. Cheng Peng and Ling Huang for the work in early literature screening.

\section{Statement of Ethics}

An ethics statement is not applicable because this study is based exclusively on published literature.

\section{Conflict of Interest Statement}

The authors have no conflicts of interest to declare.

\section{Funding Sources}

No funding was received for this research.

\section{Author Contributions}

J.F.L., J.L.H., and D.R.H. contributed to study conception. J.F.L., D.R.H., J.L.H., X.C., Y.J.F., C.W., and M.A.A. contributed to data collection. J.F.L. and D.R.H. contributed to data analysis. J.F.L. and D.R.H. contributed to manuscript writing. D.R.H., Y.W., and Y.T. contributed to critical revision. J.F.L., D.R.H., J.L.H., X.C., Y.J.F., C.W., and M.A.A. contributed to the final approval.

\section{Data Availability Statement}

All data generated or analyzed during this study are included in this article (and/or) its supplementary material files. Further inquiries can be directed to the corresponding author. 


\section{References}

1 Macdonald RL, Schweizer TA. Spontaneous subarachnoid haemorrhage. Lancet. 2017; 389:655-66.

2 De Rooij NK, Linn FH, van der Plas JA, Algra A, Rinkel GJ. Incidence of subarachnoid haemorrhage: a systematic review with emphasis on region, age, gender and time trends. J Neurol Neurosurg Psychiatry. 2007;78: 1365-72.

3 Macdonald RL. Delayed neurological deterioration after subarachnoid haemorrhage. Nat Rev Neurol. 2014;10:44-58.

4 Dorhout Mees SM, Rinkel GJ, Feigin VL, Algra $A$, van den Bergh WM, Vermeulen M, et al. Calcium antagonists for aneurysmal subarachnoid haemorrhage. Cochrane Database Syst Rev. 2007;2007(3):CD000277.

5 Daou BJ, Koduri S, Thompson BG, Chaudhary N, Pandey AS. Clinical and experimental aspects of aneurysmal subarachnoid hemorrhage. CNS Neurosci Ther. 2019;25:1096112 .

6 Nishino A, Umegaki M, Fujinaka T, Yoshimine T. Cilostazol attenuates cerebral vasospasm after experimental subarachnoid hemorrhage. Neurol Res. 2010;32:873-8.

7 Yamaguchi-Okada M, Nishizawa S, Mizutani A, Namba H. Multifaceted effects of selective inhibitor of phosphodiesterase III, cilostazol, for cerebral vasospasm after subarachnoid hemorrhage in a dog model. Cerebrovasc Dis. 2009;28:135-42.

8 Ito H, Fukunaga M, Suzuki H, Miyakoda G, Ishikawa M, Yabuuchi Y, et al. Effect of cilostazol on delayed cerebral vasospasm after subarachnoid hemorrhage in rats: evaluation using black blood magnetic resonance imaging. Neurobiol Dis. 2008;32:157-61.

9 Yoshimoto T, Shirasaka T, Fujimoto S, Yoshidumi T, Yamauchi T, Tokuda K, et al. Cilostazol may prevent cerebral vasospasm following subarachnoid hemorrhage. Neurol Med Chir. 2009;49:235-40.

10 Suzuki S, Sayama T, Nakamura T, Nishimura $\mathrm{H}$, Ohta $\mathrm{M}$, Inoue $\mathrm{T}$, et al. Cilostazol improves outcome after subarachnoid hemorrhage: a preliminary report. Cerebrovasc Dis. 2011;32: 89-93.

11 Senbokuya N, Kinouchi H, Kanemaru K, Ohashi Y, Fukamachi A, Yagi S, et al. Effects of cilostazol on cerebral vasospasm after aneurysmal subarachnoid hemorrhage: a multicenter prospective, randomized, open-label blinded end point trial. J Neurosurg. 2013; 118:121-30.

12 Kimura H, Okamura Y, Chiba Y, Shigeru M, Ishii T, Hori T, et al. Cilostazol administration with combination enteral and parenteral nutrition therapy remarkably improves outcome after subarachnoid hemorrhage. Acta Neurochir Suppl. 2015;120:147-52.

13 Matsuda N, Naraoka M, Ohkuma H, Shimamura N, Ito K, Asano K, et al. Effect of cilostazol on cerebral vasospasm and outcome in patients with aneurysmal subarachnoid hemorrhage: a randomized, double-blind, placebo-controlled trial. Cerebrovasc Dis. 2016;42:97-105.

14 Nakatsuka Y, Kawakita F, Yasuda R, Umeda Y, Toma N, Sakaida H, et al. Preventive effects of cilostazol against the development of shunt-dependent hydrocephalus after subarachnoid hemorrhage. J Neurosurg. 2016; 127:319-26.

15 Sugimoto K, Nomura S, Shirao S, Inoue T, Ishihara $\mathrm{H}$, Kawano R, et al. Cilostazol decreases duration of spreading depolarization and spreading ischemia after aneurysmal subarachnoid hemorrhage. Ann Neurol. 2018;84: 873-85.

16 Suzuki H, Nakatsuka Y, Yasuda R, Shiba M, Miura Y, Terashima M, et al. Dose-dependent inhibitory effects of cilostazol on delayed cerebral infarction after aneurysmal subarachnoid hemorrhage. Transl Stroke Res. 2019;10: 381-8.

17 Zheng H, Yang H, Gong D, Mai L, Qiu X, Chen L, et al. Progress in the mechanism and clinical application of cilostazol. Curr Top Med Chem. 2019;19:2919-36.

18 Niu PP, Yang G, Xing YQ, Guo ZN, Yang Y. Effect of cilostazol in patients with aneurys- mal subarachnoid hemorrhage: a systematic review and meta-analysis. J Neurol Sci. 2014; 336:146-51.

19 Saber H, Desai A, Palla M, Mohamed W, Seraji-Bozorgzad N, Ibrahim M. Efficacy of cilostazol in prevention of delayed cerebral ischemia after aneurysmal subarachnoid hemorrhage: a meta-analysis. J Stroke Cerebrovasc Dis. 2018;27:2979-85.

20 Shan T, Zhang T, Qian W, Ma L, Li H, You C, et al. Effectiveness and feasibility of cilostazol in patients with aneurysmal subarachnoid hemorrhage: a systematic review and metaanalysis. J Neurol. 2020;267:1577-84.

21 Higgins JP, Altman DG, Gøtzsche PC, Jüni P, Moher D, Oxman AD, et al. The Cochrane collaboration's tool for assessing risk of bias in randomised trials. BMJ. 2011;343:d5928.

22 Stang A. Critical evaluation of the NewcastleOttawa scale for the assessment of the quality of nonrandomized studies in meta-analyses. Eur J Epidemiol. 2010;25:603-5.

23 Higgins JP, Thompson SG, Deeks JJ, Altman DG. Measuring inconsistency in meta-analyses. BMJ. 2003;327:557-60.

24 Suzuki H, Kawakita F. Tenascin-C in aneurysmal subarachnoid hemorrhage: deleterious or protective? Neural Regen Res. 2016;11: 230-1.

25 Suzuki H, Fujimoto M, Kawakita F, Liu L, Nakatsuka Y, Nakano F, et al. Tenascin-C in brain injuries and edema after subarachnoid hemorrhage: findings from basic and clinical studies. I Neurosci Res. 2020;98:42-56.

26 Suzuki H, Nishikawa H, Kawakita F. Matricellular proteins as possible biomarkers for early brain injury after aneurysmal subarachnoid hemorrhage. Neural Regen Res. 2018;13: $1175-8$

27 Mutoh T, Kazumata K, Yokoyama Y, Ishikawa T, Taki Y, Terasaka S, et al. Comparison of postoperative volume status and hemodynamics between surgical clipping and endovascular coiling in patients after subarachnoid hemorrhage. J Neurosurg Anesthesiol. 2015 Jan;27(1):7-15. 